\title{
Interdisciplinary treatment of the patient with adenoid cystic carcinoma of the Bartholin's gland resulting in 15 years' survival: a case report and review of literature
}

\author{
Marek Nowak ${ }^{1}$, Magdalena Rycel $^{1}$, Marian Szpakowski $^{1}$, Andrzej Kulig ${ }^{2}$, Janusz Sobotkowski ${ }^{3}$, Adam Dziki $^{4}$ \\ ${ }^{1}$ Department of Gynecology and Gynecologic Oncology, Polish Mother's Memorial Hospital - Research Institute, Lodz, Poland \\ 2Department of Pathology, Polish Mother's Memorial Hospital - Research Institute, Lodz, Poland \\ ${ }^{3}$ Department of Brachytherapy, Regional Oncological Centre, Lodz, Poland \\ ${ }^{4}$ Department of General and Colorectal Surgery, Medical University of Lodz, Poland
}

\begin{abstract}
Carcinoma of the Bartholin's gland is very rare, comprises below 2\% of Bartholin's gland lesions and adenoid cystic carcinoma (ADC) is one of the most uncommon variants and accounts for 10-15\% of Bartholin's gland malignancies. There is no consensus on treatment of ADC of the Bartholin's gland: reported cases were treated with local excision or vulvectomy with or without lymphadenectomy followed or not by radiotherapy. The survival of patients varies significantly, so we present a case of interdisciplinary treatment of ADC resulting in 15 years' survival. The patient was initially treated with local excision, but the margins were not clear. Then vulvectomy, inguinal lymphadenectomy and adjuvant brachytherapy were performed resulting in 7 years free of the disease. Relapses were excised by abdominoperineal amputation of the rectum and distal part of the vagina with sigmoideostomy, excisions of local recurrences in vagina and metastasectomy of isolated lung metastases. The patient died manifesting multiple lung metastases 15 years after the initial diagnosis. Based on our experience and world literature, in cases of adenoid cystic carcinoma of the Bartholin's gland, vulvectomy with or without lymphadenectomy should be considered as a treatment of choice and in patients with positive margin, surgery should be extended by adjuvant radiotherapy.
\end{abstract}

Key words: adenoid cystic carcinoma, Bartholin's gland, vulvectomy.

\section{Introduction}

Carcinoma of the Bartholin's gland is very rare and comprises below $2 \%$ of Bartholin's gland lesions and less than $1 \%$ of neoplasms of the female genital tract $[1,2]$. Adenoid cystic carcinoma (ADC) is one of the most uncommon variants and accounts for $10-15 \%$ of Bartholin's gland malignancies; to date only about 80 cases have been reported in the literature $[3,4]$. There is no consensus on treatment of ADC of Bartholin's gland. Reported cases were treated with local excision or vulvectomy with or without lymphadenectomy followed or not by radiotherapy $[3,5]$. Thus, the survival of patients varies significantly, but generally most of them died before 5 years, although there were a few patients surviving 10 to even 20 years [3].

There is no consensus on treatment of adenoid cystic carcinoma (ADC) of the Bartholin's gland, so we present a case of interdisciplinary treatment of ADC resulting in 15 years' survival with a review of the literature.

\section{Case report}

At the end of 1993, a 44-year-old woman presented the feeling of compression in the vulvar region radiating to the left thigh, initially treated as inflammation of the left Bartholin's gland. After 3 months, a small tumor (3 cm in diameter) in the left labia majora was palpated and excised. Histopathologic examination showed adenoid cystic carcinoma - incomplete excision (Fig. 1). Consecutive bilateral inguinal lymphadenectomy with radicalization of local excision revealed no metastases in lymph nodes and adenoid cystic carcinoma with low atypia and mitotic activity but invading stroma and spreading along the nerves in the perineural space - and still incomplete excision. After this, partial vulvectomy was performed resulting in excision of the disease with focus of carcinoma localized close to the surgical incision. Adjuvant radium brachytherapy of 24 mcd was given. The patient was continuously monitored with clinical examinations, CT scans, endoscopies of the rectum and colon (there were periodical bleed-

Corresponding author:

Marek Nowak, Department of Gynecology and Gynecologic Oncology, Polish Mother's Memorial Hospital - Research Submitted: 6.06.2014 Institute, 281/289 Rzgowska St., 93-338 tódź, Poland, phone: +48 4227111 59, e-mail: mrn@poczta.onet.pl Accepted: 11.09 .2014 
ings from the rectum) and was free of the disease for 7 years. At the end of 2001, acute vulvar pain radiating to the vagina and rectum appeared. Three biopsies from the rear frenulum and the left part of vaginal vestibule showed the recurrence of adenoid cystic carcinoma. Abdominoperineal amputation of the rectum and distal part of the vagina with sigmoideostomy was performed. Histopathologic exam showed adenoid cystic carcinoma focally differentiating towards mixed malignant tumor - surgical stumps were free of neoplasia. In 2002, a small lesion of the right labia majora occurred and was excised and histopathologically confirmed as metastasis of previously operated carcinoma of the left Bartholin's gland. In 2005, local recurrence in the right part of the anterior vaginal wall was excised. In January 2008, there was metastasectomy of isolated lung metastases. In May 2009, the patient died manifesting multiple lung metastases.

\section{Discussion}

Due to the fact that ADC of the Bartholin's gland accounts for less than $1 \%$ of all genital tract neoplasms, all reports on this subject are based on descriptions of individual cases. The age distribution of patients with ADC of Bartholin's gland shows no particular pattern, but generally it occurs in middle-aged and older women. The symptoms and first signs of this neoplasm are usually non-specific. They may include inflammation, pain, burning sensation, bleeding, dyspareunia and/or palpable mass of labia majora [1, 3]. Thus, in the first step of clinical diagnosis it is sometimes difficult to differentiate mild inflammation, abscess and cysts of the Bartholin's gland from neoplastic tumors. Due to diag nostic problems patients are often exposed to multiple operations: initially conducted marsupialization or cystectomy is usually correlated with lack of appropriate margin free of carcinoma and necessity of repeating the surgical treatment with margin tissue excision or vulvectomy. We dealt with similar diagnostic difficulties in

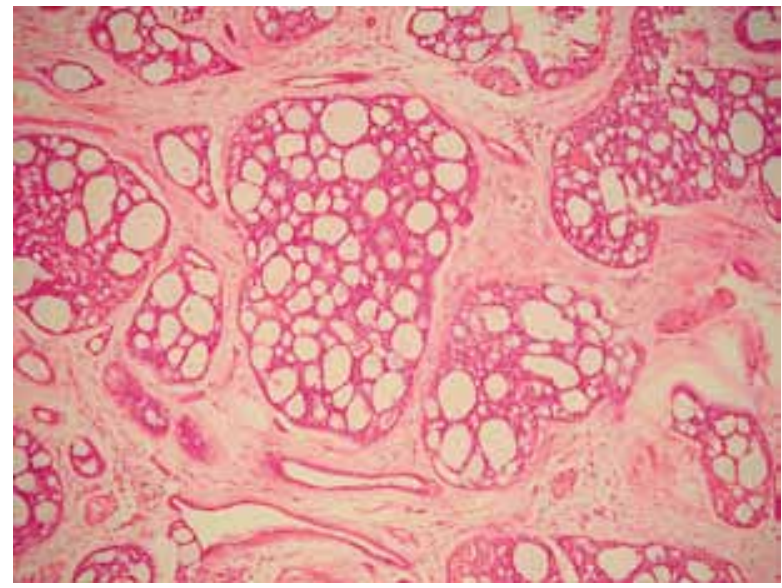

Fig. 1. Adenoid cystic carcinoma of Bartholin's gland (HE, 100×) the case of the described patient. During the first visit she presented non-specific clinical symptoms and was initially treated for inflammation of Bartholin's gland. Also the nodule which appeared in vulva 3 months after the beginning of the inflammation, did not awaken the suspicion of malignant tumor and therefore it was cut off without the appropriate tissue margin. As a result, only after the histopathological examination of the excised tumor the patient was qualified for bilateral lymphadenectomy with radicalization of local excision. Because of these reasons doctors should be especially suspicious when dealing with patients over 40 years old when they observe a homogenous vulvar lesion.

On the basis of reported cases of patients from all over the world we recognize two procedure models of treatment depending on the patients' clinical state: local excision of the cancer or radical vulvectomy with or without lymphadenectomy. As far as our experience is concerned, local excision only is neither a sufficient nor recommended procedure to protect the patient from the relapse of the cancer, especially when removing the tumor within healthy tissue margin was unsuccessful. This is confirmed by the data provided by Anaf et al. [5]. In a group of 43 patients with confirmed ADC in histopathology, they observed that local excision carried a higher risk of relapse (in $43 \%$ of patients) compared to patients after radical vulvectomy (relapse in 9\%) [5]. These data are also supported by the case of our patient, in whom we observed 7 years free of the disease since vulvectomy with lymphadenectomy until the relapse. Similar results were showed by Alsan Cetin et al. [3]. Relapse was observed in $26 \%$ of patients after local excision and in $7 \%$ after vulvectomy [3].

Despite inguinal lymphadenectomy carried out in our patient and obtaining the desired clinical outcome, we have not found in the literature enough scientific information allowing us to formulate an un-

Tab. I. The treatment and clinical outcome of patients with adenoid cystic carcinoma of Bartholin's gland; summary includes only reports with follow up after treatment [3-5 and present case]

\begin{tabular}{lcc}
\hline Treatment & $\begin{array}{c}\text { Margins } \\
n(\%)\end{array}$ & $\begin{array}{c}\text { Clinical outcome* }(\mathrm{yr}) \\
\text { mean } \pm \text { SD (range) }\end{array}$ \\
\hline $\mathrm{LE}$ & $\mathrm{N}-8(80 \%)$ & $\mathrm{NED}-4.0 \pm 3.5(0.8-12)(n=11)$ \\
$(n=11)$ & $\mathrm{P}-2(20 \%)$ & \\
\hline $\mathrm{LE}+\mathrm{RT}$ & $\mathrm{N}-3(37.5 \%)$ & $\mathrm{NED}-9.0 \pm 7.8(2.7-20)(n=4)$ \\
$(n=11)$ & $\mathrm{P}-5(62.5 \%)$ & $\mathrm{D}-9.7 \pm 8.2(2-26)(n=7)$ \\
\hline $\mathrm{V}$ & $\mathrm{N}-9(64.3 \%)$ & $\mathrm{NED}-2.2 \pm 1.8(0.7-6)(n=10)$ \\
$(n=15)$ & $\mathrm{P}-5(35.7 \%)$ & $\mathrm{D}-7.2 \pm 4.9(3-13)(n=5)$ \\
\hline $\mathrm{V}+\mathrm{RT}$ & $\mathrm{N}-5(62.5 \%)$ & $\mathrm{NED}-5.9 \pm 5.8(1-15)(n=7)$ \\
$(n=9)$ & $\mathrm{P}-3(37.5 \%)$ & $\mathrm{D}-5.1 \pm 0.2(5-5.25)(n=2)$ \\
\hline
\end{tabular}

$\mathrm{LE}$ - local excision, $\mathrm{V}$ - vulvectomy, $\mathrm{RT}$ - radiotherapy, $\mathrm{N}$ - negative, $\mathrm{P}$ - positive

Clinical outcome* - follow-up of previously presented cases varied significantly from 7 months to 26 years and the end points usually were: time with no evidence of disease (NED) or death (D) 
ambiguous conclusion as to the necessity of routine lymphadenectomy in all patients suffering from ADC of Bartholin's gland. The research conducted by Anaf et al. [5] confirmed it, as in only 2 cases out of 22 patients with performed lymphadenectomy, metastases to lymph nodes occurred. Scientists are favorable to the possibility of using routine radiotherapy in treating ADC of Bartholin's gland. Patients, in whom adjuvant radiotherapy was used, lived longer and there were fewer cases of local relapses as well as distant metastases [3]. In the group of patients with ADC, distant metastases occurred in bones and lungs [3]. Distant metastases were observed two times more often in patients with positive tissue margins on histopathology than in cases of negative margins [6]. In patients with confirmed multiple distant metastases, chemotherapy seems to be the method of choice, but there is no reported material or clinical research assessing the efficiency of this method of treatment.

Adenoid cystic carcinoma of Bartholin's gland is considered to be a tumor of relatively slow growth but it is very aggressive locally with a high tendency to create local relapses. The average survival of patients with ADC of Bartholin's gland is assessed to be 5 to 10 years [7]. Overall survival varies significantly according to the clinical advancement of the disease and is shorter in patients with positive tissue margins (below 7 years) whereas in cases of negative margins it is prolonged to 15 and even 20-30 years [3]. The methods of treatment and clinical outcome of the reported cases of ADC of Bartholin's gland are summarized in Table I.

\section{Conclusions}

On the basis of our own experience and world literature information, in the group of patients with adenoid cystic carcinoma of the Bartholin's gland vulvectomy with or without lymphadenectomy should be considered as a treatment of choice and in patients with positive margin, surgery should be extended by adjuvant radiotherapy. Such treatment is advisable because of the longer survival, despite the load and degree of the patient's mutilation connected with vulvectomy compared to the local excision of tumor.

\section{Disclosure}

Authors report no conflicts of interest.

\section{References}

1. Cardosi RJ, Speights A, Fiorica JV, et al. Bartholin's gland carcinoma: a 15-year experience. Gynecol Oncol 2001; 82: 247-251.

2. Sośnik H, Sośnik K, Hałoń A. The patomorphology of Bartholin's gland. Analysis of surgical data. Pol J Pathol 2007; 58: 99-103.

3. Alsan Cetin I, Vinh-Hung V, Eren F, Abacioglu U. Adenoid cystic carcinoma of the Bartholin's gland: case report and systematic review of the literature. Eur J Gynecol Oncol 2011; 32: 567-572.

4. Khanna G, Rajni, Azad K. Bartholin gland carcinoma. Indian J Pathol Microbiol 2010; 53: 171-172.

5. Anaf V, Buxant F, Rodesh F, et al. Adenoid cystic carcinoma of Bartholin's gland: what is the optimal approach? Eur J Surg Oncol 1999; 25 : 406-409.

6. Dodson MG, O'Leary JA, Orfei E. Adenoid cystic carcinoma of the vulva. Malignant cylindroma. Obstet Gynecol 1978; 51: 26-29.

7. Woida FM, Ribeiro-Silva A. Adenoid cystic carcinoma of the Bartholin gland. Arch Pathol Lab Med 2007; 131: 796-798. 